\title{
Efficacy and Safety of Tofacitinib for Treatment of Moderate to Severe Active Ulcerative Colitis: First Report from Iran
}

\author{
Maryam Jameshorani, MD; Homayoon Vahedi, MD²; Anahita Sadeghi, MD²; Ali Reza Sima, MD²; Amir Anushiravani, MD²; Helia \\ Nateghi Beige, MSc²; Masoud M. Malekzadeh, MD²; Shokoofeh Naserinejad, MSc'; Sudabeh Alatab, MD, PhD ${ }^{2 *}$ \\ ${ }^{1}$ Department of Internal Medicine, School of Medicine, Metabolic Disease Research Center, Zanjan University of Medical Sciences, \\ Zanjan, Iran \\ ${ }^{2}$ Digestive Disease Research Center, Digestive Disease Research Institute, Tehran University of Medical Sciences, Tehran, Iran
}

\begin{abstract}
Background: Tofacitinib, a selective inhibitor of JAK/STAT pathway, has recently become available in our region. Here, we examined the safety and efficacy of tofacitinib in active ulcerative colitis (UC).

Methods: In a prospective, non-randomized, placebo-free, 52-week clinical trial defined in two phases of induction and maintenance, adult patients with active UC and no response or loss of response to previous conventional treatments, or anti-TNF were recruited (IRCT20181217042020N2). Patients received $10 \mathrm{mg} / \mathrm{BID}$ of tofacitinib for 8 weeks. Clinically responding patients were entered into the maintenance phase and received tofacitinib $5 \mathrm{mg} / \mathrm{BID}$ for 44 weeks. Clinical evaluation, biochemical tests and endoscopy at time points of baseline, 8, 24 and 52 weeks were performed. The primary outcome was clinical remission at 8 and 52 weeks.

Results: Fifty out of 53 enrolled patients completed the induction phase. Clinical response and clinical remission at 8 weeks occurred in $84 \%$ and $9.5 \%$, respectively. Forty-two patients who had clinical response entered the maintenance phase. Clinical remission based on the total Mayo score and the partial Mayo score occurred in $38.9 \%$ and $55.3 \%$ at 24 weeks and in $61.1 \%$ and $72.2 \%$ at 52 weeks, respectively. There was significant correlation between the total and partial Mayo score with regard to clinical remission in both 24 and 52 weeks. No serious adverse events, no case of herpes zoster, but two cases of deep vein thrombosis were seen.

Conclusions: Our study showed acceptable efficacy and safety for tofacitinib and suggested a correlation between the total Mayo score with partial Mayo score with regard to clinical remission.

Keywords: Inflammatory bowel disease, Therapy, Ulcerative colitis

Cite this article as: Jameshorani M, Vahedi H, Sadeghi A, Sima AR, Anushiravani A, Nateghi Beige H, et al. Efficacy and safety of tofacitinib for treatment of moderately to severely active ulcerative colitis: first report from Iran. Arch Iran Med. 2021;24(5):354363. doi: 10.34172/aim.2021.52
\end{abstract}

Received: July 28, 2020, Accepted: February 27, 2021, ePublished: May 1, 2021

\section{Introduction}

Inflammatory bowel disease (IBD), a chronic autoimmune disorder of the gastrointestinal tract, is typically classified into two main subtypes of ulcerative colitis (UC) and Crohn's disease (CD). The recent published data based on the Global Burden of Disease showed that 6.8 million people are suffering from this disease around the world, ${ }^{1}$ a fact that makes IBD a global disease in the $21^{\text {st }}$ century. Although IBD was believed to be a Western disease, recent data shows an increasing trend in regions such as Asia and Africa that were traditionally known as low-prevalence regions. ${ }^{2}$ A recent nation-wide report in our country also showed an increasing trend in both incidence and prevalence of IBD. ${ }^{3}$

Patients with UC, the more common subtype of IBD, might present with intermittent episodes of disease activation with symptoms of rectal bleeding, diarrhea, abdominal cramps, urgency or tenesmus, and fever. ${ }^{4}$ The mucosal inflammation with a relapsing and remitting nature usually starts in the rectum and extends to proximal segments of the colon. ${ }^{4}$ While the initial mechanisms underlying the highly complex pathogenesis of the disease are still under debate, the crucial role of inflammatory cytokines in controlling intestinal inflammation and the associated clinical symptom, once the disease is established, has been well documented and monoclonal antibodies targeting key cytokines (for example, TNF) are frequently used for treatment of patients with refractory UC. ${ }^{5,6}$ Newer therapy approaches are now relying on interfering with cytokine signaling events, including those dependent on the Janus kinase (JAK) family of tyrosine kinases and the signal transducer and activator of transcription (STAT) family of DNA-binding proteins. ${ }^{6}$ In vivo and clinical studies showed lines of evidence indicating that JAK-STAT signaling is required to mediate the cytokine function and that JAK inhibitors can block 
the activity of multiple cytokines and cytokine pathways simultaneously. ${ }^{7-8}$ In fact, genome-wide association studies have shown that JAK2-IL-23, SMAD3 and IL2RA pathways are associated with IBD susceptibility loci and that patients with IBD might benefit from therapies against these molecular targets. ${ }^{9-11}$ These confirmations have led to the development of a pan-JAK inhibitor, named tofacitinib, for the treatment UC. Tofacitinib is an orally available, small-molecule inhibitor of JAK1 and JAK3, as well as, to a lesser extent, JAK2 and TYK2 enzymes. JAK1/JAK3 dimerization can control signaling of many cytokines including IL-2, IL-4, IL-7, IL-9, IL-15, and IL-21 many of which have an important role in UC pathogenesis. $^{6}$

Tofacitinib has been approved for treatment of rheumatoid arthritis and moderately to severely active UC. Its safety and efficacy in induction and maintenance therapy compared to placebo in patients with moderately to severely active UC have been demonstrated in a phase II trial, ${ }^{12}$ and three phase III trials with tofacitinib (OCTAVE Induction 1 and 2 and OCTAVE Sustain). ${ }^{13}$ Employment of this agent for treatment of UC in our region is novel and no study has been done so far to evaluate the efficacy of this medication. In this study, we conducted a clinical trial and we report the efficacy and safety of tofacitinib (Iranian generic) as induction and maintenance therapy for moderately to severely active UC.

\section{Materials and Methods}

This prospective study was a single-center, 52-week, openlabel, non-randomized, phase II trial to evaluate the safety and efficacy of tofacitinib for induction (induction study) and maintenance (maintenance study) therapy in adults with moderately to severely active UC.

The study was conducted in the Digestive Disease Research Institute of Tehran University of Medical Sciences, Tehran, Iran. The study was conducted from November 2018 to March 2020. The patients enrolled using the Iranian Registry of Crohn's and Colitis (IRCC). The demographic and anthropometric data of patients were collected by means of a questionnaire using the IRCC. Full colonoscopy was performed by the central team for assessment of disease extension and also ruling out Crohn's disease.

\section{Patient Selection}

Adults aged 18 to 80 years were eligible for inclusion in the trial if they had a confirmed diagnosis of UC for at least 3 months before enrollment; had moderately to severely active UC, defined as a total score of 5 to 12 on the Mayo score (total Mayo scores range from 0 to 12, with higher scores indicating more severe disease) ${ }^{14}$ and a subscore of at least 2 on the endoscopic component of the Mayo score and a rectal bleeding subscore of at least 1 (subscores on each of the four components of the Mayo score range from 0 to 3); had at least proctosigmoiditis; and had no response or loss of response or unacceptable side effects to previous conventional treatments (including glucocorticoids, azathioprine, mercaptopurine), or Anti-TNF therapy. Patients were excluded from study if they had clinical findings suggestive of $\mathrm{CD}$; history of $\mathrm{CD}$ (Crohn's colitis, or ileo-colitis), clinical signs of fulminant colitis, toxic megacolon, or microscopic colitis, indeterminate colitis, infectious colitis, or ischemic colitis; had a confirmed cancer; and were pregnant or intended to become pregnant.

Concomitant consumption of oral aminosalicylates was allowed if they were administered in a stable dose during the trial period. Concomitant consumption of oral glucocorticoids was allowed only with mandatory tapering. Concomitant consumption of TNF antagonists, azathioprine, methotrexate, and mercaptopurine was prohibited. Patients receiving azathioprine, 6-mercaptopurine or methotrexate were required to stop these agents at least two weeks before enrollment. Patients receiving TNF antagonist were required to stop these agents at least 8 weeks before enrollment.

Potential participants underwent preliminary viral screening tests including: Anti HCV Ab, Anti HIV, HBS $\mathrm{Ag}$, Anti HBC Ab and Anti HBS Ab, and interferon- $\gamma$ release assays for ruling out tuberculosis and those found to be eligible were invited to attend a baseline assessment. Patients were also provided with the physician's contact details to discuss any concerns during the study.

\section{Study Design}

The study comprised a screening visit within 4 weeks of baseline, 8 weeks of induction period followed by 44 weeks of maintenance period. Figure 1 presents the detailed study design. In induction therapy, eligible patients received tofacitinib (Iranian generic) $10 \mathrm{mg}$ BID for 8 weeks. ${ }^{12}$ Patients who completed the induction period and achieved a clinical response or clinical remission ${ }^{12}$ with no serious adverse events following treatment, were eligible to enter the maintenance study. Patients who did not respond at week 8 were discontinued.

In the maintenance study, patients received tofacitinib $5 \mathrm{mg}$ BID for 44 weeks. ${ }^{13}$ An obligatory steroid taper algorithm was applied for patients receiving oral corticosteroids, starting at baseline of the induction therapy with the dose decreased by $5 \mathrm{mg}$ prednisolone per week until the dose reached $20 \mathrm{mg} / \mathrm{d}$ and then $2.5 \mathrm{mg} /$ wk until discontinuation. Patients who experienced recurrence of clinical symptoms following corticosteroid tapering were allowed to increase the dose back to their baseline and tapering schedule was repeated within 2 weeks. Patients who were corticosteroid dependent were excluded from the study.

The Mayo score was determined at baseline, 8, 24, and 52 weeks. The partial Mayo score (based on three of the four questions, without endoscopic subscore, range 0 to 9) was determined at $0,8,24$ and 52 weeks. The patient-reported outcomes (based on two questions of stool frequency and rectal bleeding, range 0-6) was 


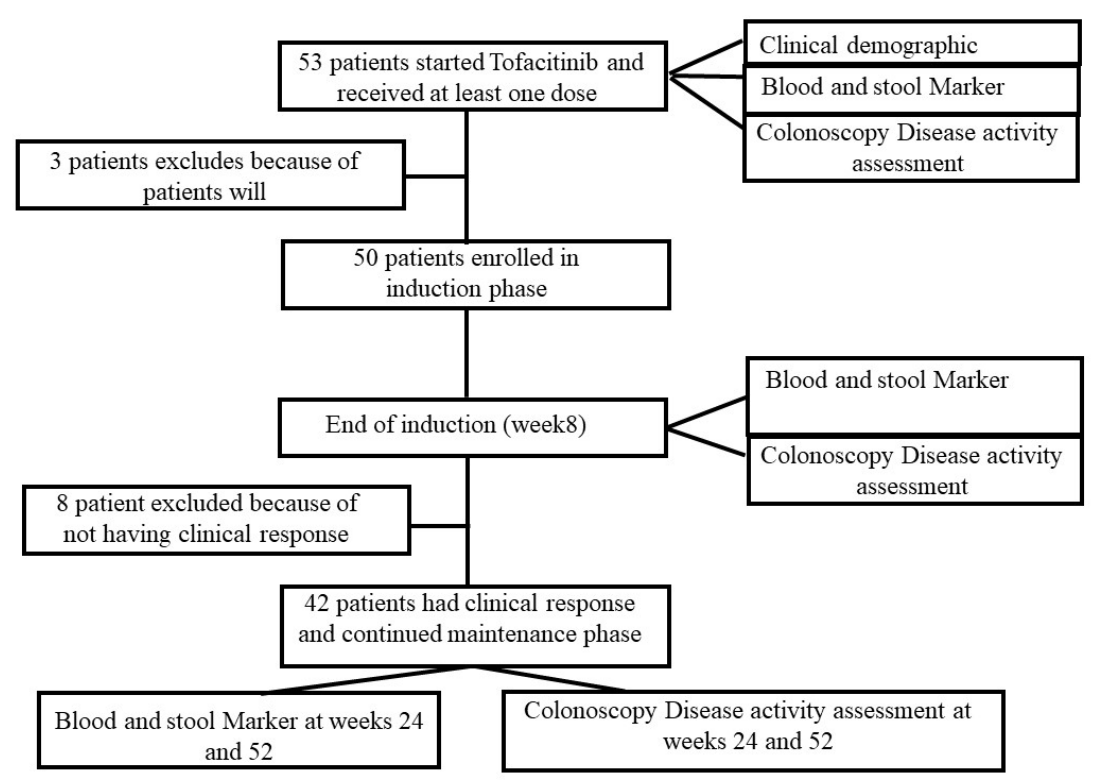

Figure 1. Flow Chart of Patients Included in the Study.

determined at $0,8,24$ and 52 weeks. For scoring rectal bleeding and stool frequency, the average subscore from the most recent consecutive 3 days before the study visit was used. Laboratory tests including lipid profile, liver and kidney functions, erythrocyte sedimentation rate (ESR) and C-reactive protein (CRP), stool OB and OP, and fecal calprotectin were measured at baseline, 8, 24 and 52 weeks. Concomitant medications and adverse events were recorded throughout all the study period.

\section{Definitions}

Clinical response: an absolute reduction in total Mayo score of $\geq 3$ points and $\geq 30 \%$ from baseline with an accompanying decrease in rectal bleeding subscore of $\geq 1$ point or absolute rectal bleeding subscore of $\leq 1$.

Clinical remission based on complete Mayo score: total Mayo score of $\leq 2$ and no individual subscore $>1$.

Clinical remission based on partial Mayo score: partial Mayo score of $\leq 2$ and no individual subscore $>1$.

Clinical remission based on patient reported outcome: subscore $=0$ in both rectal bleeding and stool frequency

Endoscopic mucosal healing: Mayo endoscopic subscore $\leq 1$.

Secondary loss of response: either clinical exacerbation following initial clinical response, need for surgery or initiation of corticosteroids.

\section{Efficacy and Safety}

Patients eligible to enter the study were assessed for all clinical outcomes at weeks 8, 24 and 52 .

The primary outcome of the tofacitinib therapy in this study was the proportion of patients in clinical remission, based on the complete Mayo score. ${ }^{13}$ The secondary outcomes of this study were: proportion of patients in clinical remission based on the partial Mayo score; proportion of patients in clinical remission based on patient reported outcome; proportion of patients in endoscopic mucosal healing at weeks 24 and 52; and proportion of patients with secondary loss of response.

Safety was assessed based on serious adverse events (SAEs), adverse events (AEs) defined as any AE regardless of relationship to study drug, and laboratory abnormalities according to the Common Terminology Criteria for Adverse Events (CTCAE) version 4.0. They were obtained through physical examination and biochemical assays and were recorded throughout the trial period.

\section{Statistical Analysis}

Statistical analyses of safety and efficacy data in this study are descriptive in nature. All analyses are based on observed data with no imputation for missing data.

The continuous variables are presented as mean \pm SD. The categorical variables are expressed as absolute values and frequencies. A general linear model was used to calculate repeated measures of analysis of variance (ANOVA) for functional parameters from baseline at the follow-up visits. We analyzed changes from baseline in the total Mayo score, partial Mayo score, and patient reported outcome separately for induction therapy and maintenance therapy, using ANOVA with adjustment for stratification variables. The Wilcoxon rank sum test was performed to compare continuous variables while the chi-square test or Fisher's exact test were used to compare categorical variables. The safety endpoints were described and summarized by number and percentage of adverse events. Safety data were analyzed in the full analysis set defined as all patients who entered the study. ${ }^{15}$ The distribution of baseline variables and proportion of disease activity were examined by the Mann-Whitney $\mathrm{U}$ test and chi-square test. Induction efficacy data were analyzed for all patients who finished the induction phase. Maintenance efficacy data were analyzed for all patients 
who entered into the maintenance phase. The efficacy data at the end of induction trials were used as baseline data for maintenance trials. $P<0.05$ were considered statistically significant. Analyses were performed using SPSS software (version 16.0, NY, USA).

\section{Results}

\section{Efficacy at the End of Induction Phase}

In total, 53 patients were enrolled and treated in an open-label induction phase trial; of those, three patients withdrew from the study before completing the induction phase and therefore were not included in the efficacy statistical analysis. The reason for withdrawal were: undesirable complications for two patients and loss to follow up in one patient. Of those enrolled, 29 patients (58\%) were men. The mean age of disease onset in our patients was slightly less than 30 years with the majority of patients $(72 \%)$ having had involvement of the entire colon (pancolitis) at the time of enrollment (Table 1).

Following 8 weeks of receiving tofacitinib at a dose of $10 \mathrm{mg}$ twice daily, $82 \%$ of our patients had a clinical response (41 patients) and therefore continued with the maintenance therapy. Moreover, two patients who had partial clinical response continued to receive $10 \mathrm{mg}$ tofacitinib twice daily for another 4 weeks at the physician's discretion and then, they were re-evaluated at the end of 12 weeks. One of these patients responded at the end of 12 weeks and was therefore included in the maintenance therapy. Therefore, finally, $84 \%$ of our patients clinically responded to induction therapy. However, only 9.5\% of the patients $(n=4)$ had clinical remission at the end of induction. At the end of induction, the number of patients who had severely active UC, based on the total Mayo score, decreased from $100 \%$ to $30 \%$ (Table 2). The number of subjects with positive levels of CRP (>5 mg/ dL) decreased to $35.4 \%$. Both ESR and fecal calprotectin levels diminished significantly from baseline to the end of induction (Table 2). Figure 2 compares the severity (Figure 2A) and extend of colon involvement (Figure $2 \mathrm{~B})$ at the end of induction phase to that of baseline. We found that in addition to decrease in diseases severity (Figure 2A), the extent of colon involvement diminished following induction therapy such that the most prevalent type of colon involvement at the end of induction shifted from pancolitis to proctosigmoiditis (Figure 2B).

\section{Safety of Patients}

Of the 42 patients who entered the maintenance phase, two patients withdrew from study before completing the 52 weeks. The reason for withdrawal was the patients' decision because of undesired adverse events. We had no serious adverse events or deaths during the study period. None of our patients developed serious infection including clostridial infections, sepsis, tuberculosis or herpes zoster. Mild to moderate forms of bloating were the most commonly reported complaints (in 65\% of patients) followed by headache (15\%). A non-itching rash was reported in four patients. One patient developed severe facial acne. Repeated episodes of upper respiratory tract infection (more than 3 times per year) that lasted more than usual was reported in four patients. One patient had Entamoeba coli gastroenteritis infection manifested by bloody diarrhea and cramping at week 32 . Two patients (one in week 20 and the other in week 35, both males) presented with symptoms of deep vein thrombosis in one leg. Both patients were treated as outpatients. One patient (female) developed micro-hematuria detected on urine analysis at week 24 without any other signs including frequency or dysuria. Nephrology consultation was done for this patient but the tofacitinib treatment continued. In one patient (male), the liver enzymes increased by 5 times at week 52. In two patients (both in week 24), a $30 \%$ decrease occurred in serum HDL levels compared to baseline levels although significant changes were not

Table 1. Demographic and Baseline Characteristics of Patients Enrolled in the Study

\begin{tabular}{|c|c|}
\hline Characteristic & $N=53$ \\
\hline Age $(y)$, mean (SD) & $36.9(12.5)$ \\
\hline Sex (n [\%]), Male & $29(54.7)$ \\
\hline Marital status (n $[\%])$, Married & $34(64.1)$ \\
\hline \multicolumn{2}{|l|}{ Education } \\
\hline Illiterate (n [\%]) & $1(1.9)$ \\
\hline Primary school (n [\%]) & $3(5.7)$ \\
\hline High school (n [\%]) & $23(43.4)$ \\
\hline University (n [\%]) & $26(49)$ \\
\hline Age at disease onset (years), mean (SD) & $29.7(12.3)$ \\
\hline Duration of UC (years), mean (SD) & $7.2(4.5)$ \\
\hline Current smoker (n [\%]), Yes & $3(5.6)$ \\
\hline Family history of IBD (n [\%]), Yes & $9(17)$ \\
\hline Appendectomy (n [\%]), Yes & $2(3.8)$ \\
\hline Disease- related surgery (n [\%]), Yes & $2(3.8)$ \\
\hline Body mass index, kg/m² mean (SD) & $23.3(4.6)$ \\
\hline $\begin{array}{l}\text { Concomitant use of oral corticosteroids at Week } 0 \\
(\mathrm{n}[\%]), \text { Yes }\end{array}$ & $8(15.1)$ \\
\hline Concomitant use of 5 ASA at Week $0(n[\%])$, Yes & $49(92.5)$ \\
\hline Previous use of Anti-TNF antagonist (n [\%]), Yes & $37(69.8)$ \\
\hline \multicolumn{2}{|l|}{ Disease localization (n [\%]) } \\
\hline Proctosigmoiditis & $4(7.5)$ \\
\hline Left-sided colitis & $10(18.9)$ \\
\hline Pancolitis & $39(73.6)$ \\
\hline \multicolumn{2}{|l|}{$\begin{array}{l}\text { Clinical disease activity at baseline, based on total } \\
\text { Mayo score }(\mathrm{n}[\%])\end{array}$} \\
\hline Moderate (5-6) & $1(1.9)$ \\
\hline Severe $(\geq 7)$ & $52(98.1)$ \\
\hline \multicolumn{2}{|l|}{$\begin{array}{l}\text { Clinical disease activity at baseline, based on partial } \\
\text { Mayo score }(\mathrm{n}[\%])\end{array}$} \\
\hline Mild (2-4) & $1(1.9)$ \\
\hline Moderate (5-6) & $9(17)$ \\
\hline Severe (7-9) & $43(81.1)$ \\
\hline
\end{tabular}


Table 2. Biochemical and Clinical Parameters of Study Subjects at Baseline and End of Induction

\begin{tabular}{|c|c|c|c|}
\hline Parameters & $\begin{array}{c}\text { Baseline } \\
(n=50)\end{array}$ & $\begin{array}{l}\text { End of Induction } \\
\qquad(n=50)\end{array}$ & $P$ Value \\
\hline White blood cells $\left({ }^{*} 10^{3}\right.$ per $\left.\mu \mathrm{L}\right)$, mean $(\mathrm{SD})$ & $7464(2658)$ & $6766(2642)$ & 0.02 \\
\hline Hemoglobin (g/dL), mean (SD) & $12.7(1.7)$ & $13(1.4)$ & 0.3 \\
\hline Triglyceride (mg/dL), mean (SD) & $98.8(43.6)$ & $102.4(41.3)$ & 0.2 \\
\hline Cholesterol (mg/dL), mean (SD) & $165.3(35.1)$ & $166.4(30.4)$ & 0.7 \\
\hline High density lipoprotein (mg/dL), mean (SD) & $46.8(11.2)$ & $46.3(10.9)$ & 0.9 \\
\hline Low density lipoprotein (mg/dL), mean (SD) & $97.9(28.7)$ & $94.9(27.6)$ & 0.9 \\
\hline Aspartate aminotransferase (IU/L), mean (SD) & $19.9(11.7)$ & $20.8(8.4)$ & 0.3 \\
\hline Alanine aminotransferase (IU/L), mean (SD) & $20.4(11.9)$ & $20.1(10.8)$ & 0.9 \\
\hline Alkaline phosphatase (IU/L), mean (SD) & $200.2(74.2)$ & $197.5(78.1)$ & 0.7 \\
\hline ESR (mm/hour), mean (SD) & $24(16.9)$ & $16.1(12.5)$ & 0.01 \\
\hline CRP $(n[\%])$, positive & $30(66.7)$ & $17(35.4)$ & 0.1 \\
\hline Fecal calprotectin $(\mu \mathrm{g} / \mathrm{g})$, mean $(\mathrm{SD})$ & 1164.9 & 433.0 & \multirow{2}{*}{0.001} \\
\hline$\geq 150(\mu \mathrm{g} / \mathrm{g}),(n[\%])$ & $41(97.6)$ & $20(64.5)$ & \\
\hline Total Mayo score, mean (min-max) & $10.1(6-12)$ & $5.4(0-11)$ & 0.001 \\
\hline Partial Mayo score, mean (min-max) & $7.6(4-9)$ & $3.8(0-8)$ & 0.01 \\
\hline Patient-reported outcomes score, mean (min-max) & $4.96(3-6)$ & $2.3(0-5)$ & 0.01 \\
\hline
\end{tabular}

ESR, erythrocyte sedimentation rate; CRP, C-reactive protein.

Data were missing for: CRP baseline: 5 patients, CRP induction: 2 patients, fecal calprotectin baseline: 8 patients, fecal calprotectin induction: 19 patients, ESR baseline: 1 patient, ESR induction: 6 patients. The CRP level $>5 \mathrm{mg} / \mathrm{dL}$ indicated as positive CRP level.
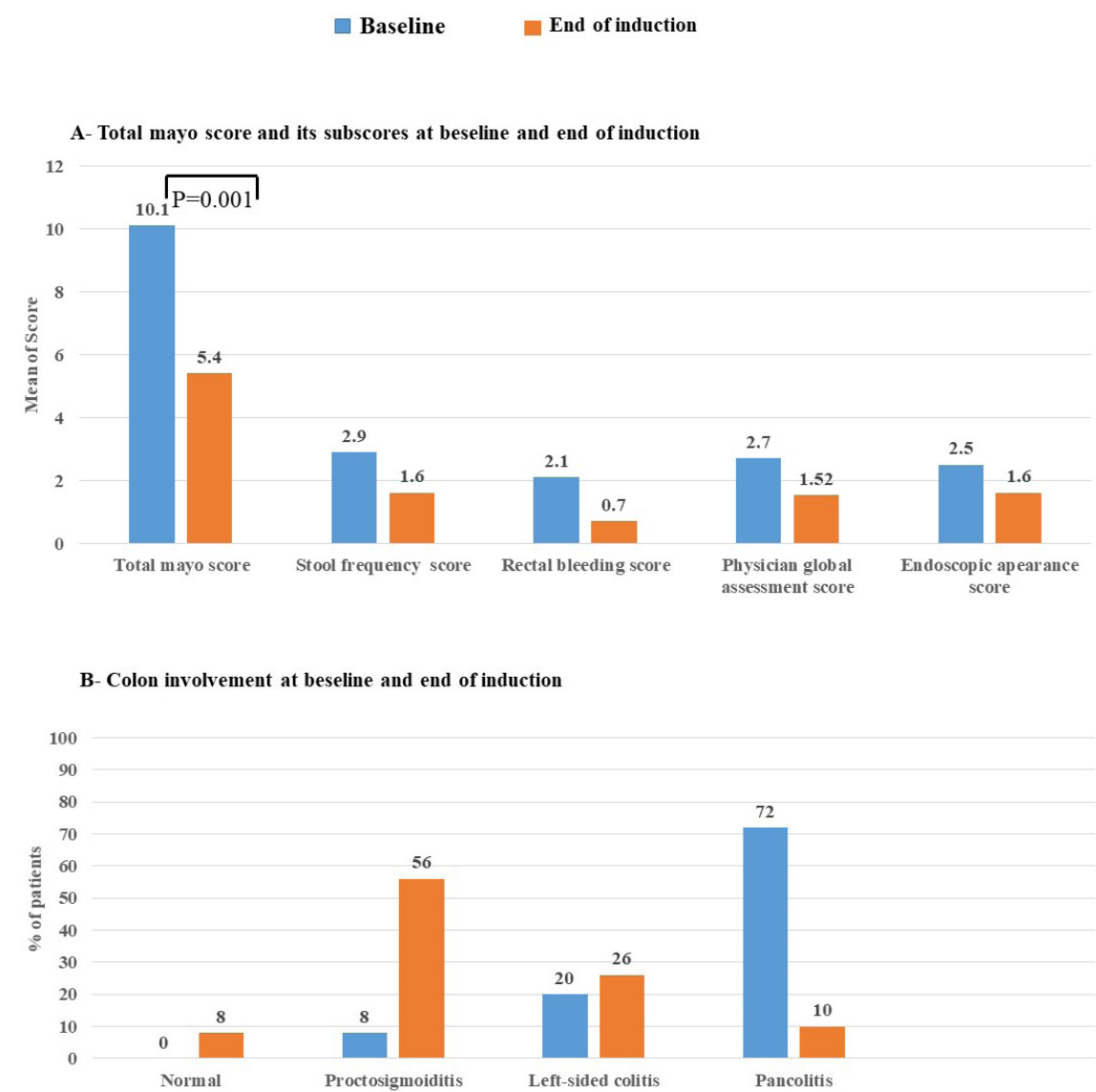

Figure 2. Comparing the Severity and Extent of Colon Involvement Following 8 Weeks of Treatment with Tofacitinib. Panel A) A significant decrease in the Mayo score (range 0-12) and its subscores (range 0-3) occurred after induction therapy. Panel B) The extent of colon involvement decreased after induction therapy. 
observed in other lipid parameters of these patients. In one patient (female), the cholesterol level increased by $45 \%$ in week 52. None of the adverse events led to permanent cessation of tofacitinib.

\section{Efficacy at the End of Maintenance Phase}

The efficacy of oral tofacitinib at a dose of $5 \mathrm{mg}$ BID in the maintenance phase was assessed by including the patients who clinically responded to this therapy in the induction phase $(n=42)$. None of the biochemical parameters, except for hemoglobin, changed significantly during the study period (Table 3 ). When we considered the clinical remission based on the total Mayo score, we found that $38.9 \%$ and $61.1 \%$ of our patients were in clinical remission at weeks 24 and 52, respectively (Table 3 ). The values for clinical remission based on the partial Mayo score at weeks 24 and 52 were $55.3 \%$ and $72.2 \%$, respectively (Figures $3 \mathrm{~A}$ and $3 \mathrm{~B})$. However, the clinical remission response based on patient-reported outcomes that included the score for both rectal bleeding and stool frequency was lower, reaching $26.2 \%$ at week 24 and $50 \%$ at week 52 . The endoscopic mucosal healing rate at week 24 was $27.8 \%$, reaching up to $44.4 \%$ at week $52(P=0.03$, week 52 vs. week 8 , Figure $3 \mathrm{~B})$. In logistic regression analysis, none of the factors of gender (being male), higher education, being married, being a smoker, having a family history of
IBD, history of appendectomy and previous use of antiTNF therapy had a significant association with clinical remission based on the total Mayo score at week 52 .

There was a significant correlation between the total Mayo score and the partial Mayo score with regard to clinical remission at weeks $24(P=0.02)$ and $52(P=0.04)$. However the correlation between the total Mayo score and patient-reported outcome with regard to clinical remission did not reach a significant level at either week 24 or 52 .

In total, secondary loss of response was observed in 23\% of our patients. In details, worsening of UC between the two time points of 8 and 24 weeks occurred in one patient (shifting from the total Mayo score category of remission to severe), while seven patients experienced recurrence of symptoms from week 8 to week 52 . For three of these seven patients, corticosteroid therapy was started, although the tapering initiated when the symptoms alleviated. On the other hand, only two patients experienced the worsening of UC score between weeks 24 and 52. One of these patients was admitted in the hospital and was a candidate for surgery; however, he refused surgery and his medication was continued of his own will.

\section{Discussion}

The worldwide published data on tofacitinib outcomes in clinical trials is sparse and more prominently missing

Table 3. Biochemical and Clinical Parameters in Subject Enrolled for Maintenance Therapy

\begin{tabular}{|c|c|c|c|c|}
\hline Parameter & $\begin{array}{l}\text { End of Induction } \\
\qquad(n=42)\end{array}$ & 24 Weeks & 52 Weeks & $P$ Value \\
\hline White blood cells (*103 per $\mu \mathrm{L})$, mean (SD) & $6838(2693)$ & $8360(10379)$ & $6582(1692)$ & 0.7 \\
\hline Hemoglobin (g/dL), mean (SD) & $12.9(1.4)$ & $13.3 *(1.9)$ & $13.6(1.3)$ & $0.006 \#$ \\
\hline Triglyceride (mg/dL), mean (SD) & $102.5(39.2)$ & $106.2(39.1)$ & $111.8(36.9)$ & 0.7 \\
\hline Cholesterol (mg/dL), mean (SD) & $166.8(27.3)$ & $178.5(38.6)$ & $178.5(31.7)$ & 0.5 \\
\hline High density lipoprotein (mg/dL), mean (SD) & $47.6(10.8)$ & $53.2(13.6)$ & $50.3(12.7)$ & 0.7 \\
\hline Low density lipoprotein (mg/dL), mean (SD) & $95.7(25.6)$ & $94.7(36.9)$ & $100(25)$ & 0.6 \\
\hline Aspartate aminotransferase (IU/L), mean (SD) & $20.4(7.5)$ & $21.1(6.5)$ & $22.5(9.2)$ & 0.1 \\
\hline Alanine aminotransferase (IU/L), mean (SD) & $19.8(10.5)$ & $22.5(9.8)$ & $22.7(14.5)$ & 0.1 \\
\hline Alkaline phosphatase (IU/L), mean (SD) & $186.5(55.2)$ & $178.7(67.8)$ & $183.6(64.8)$ & 0.9 \\
\hline ESR (mm/hour), mean (SD) & $16(12.3)$ & $12.5(8.7)$ & $15.3(13.4)$ & 0.1 \\
\hline CRP $(\mathrm{n}[\%])$, positive & $13(32.5)$ & $9(26.5)$ & $4(14.3)$ & 0.4 \\
\hline \multicolumn{5}{|l|}{ Disease localization, \% } \\
\hline Normal & 9.5 & 36.8 & 44.4 & $0.04 \#$ \\
\hline Proctosigmoiditis & 9.5 & 10.5 & 5.6 & 0.4 \\
\hline Left-sided colitis & 28.6 & 21.1 & 16.7 & 0.8 \\
\hline Pancolitis & 52.4 & 31.6 & 33.3 & 0.5 \\
\hline Fecal calprotectin $(\mu \mathrm{g} / \mathrm{g})$, mean $(\mathrm{SD})$ & $430.7(442)$ & $302(383)$ & $169.3149 .5)$ & \multirow{2}{*}{0.1} \\
\hline$\geq 150(\mu \mathrm{g} / \mathrm{g}), \%$ & 62.1 & 33.3 & 40 & \\
\hline Total Mayo score, mean (min-max) & $4.8(0-8)$ & $3.7(0-9)$ & $2.5(0-11)$ & $0.02 \#$ \\
\hline Partial Mayo score, mean (min-max) & $3.36(0-6)$ & $2.7(0-9)$ & $1.7(0-9)$ & $0.001^{*} \#$ \\
\hline Patient- reported outcomes score, mean (min-max) & $2(0-4)$ & $1.8(0-6)$ & $1.1(0-0)$ & $0.007 \#$ \\
\hline
\end{tabular}

ESR, erythrocyte sedimentation rate; CRP, C-reactive protein.

* Week 24 versus induction; \# Week 52 versus induction.

Data were missing for: Total Mayo score week 24: 24 patients, The CRP level $>5 \mathrm{mg} / \mathrm{dL}$ indicated as positive CRP level. 

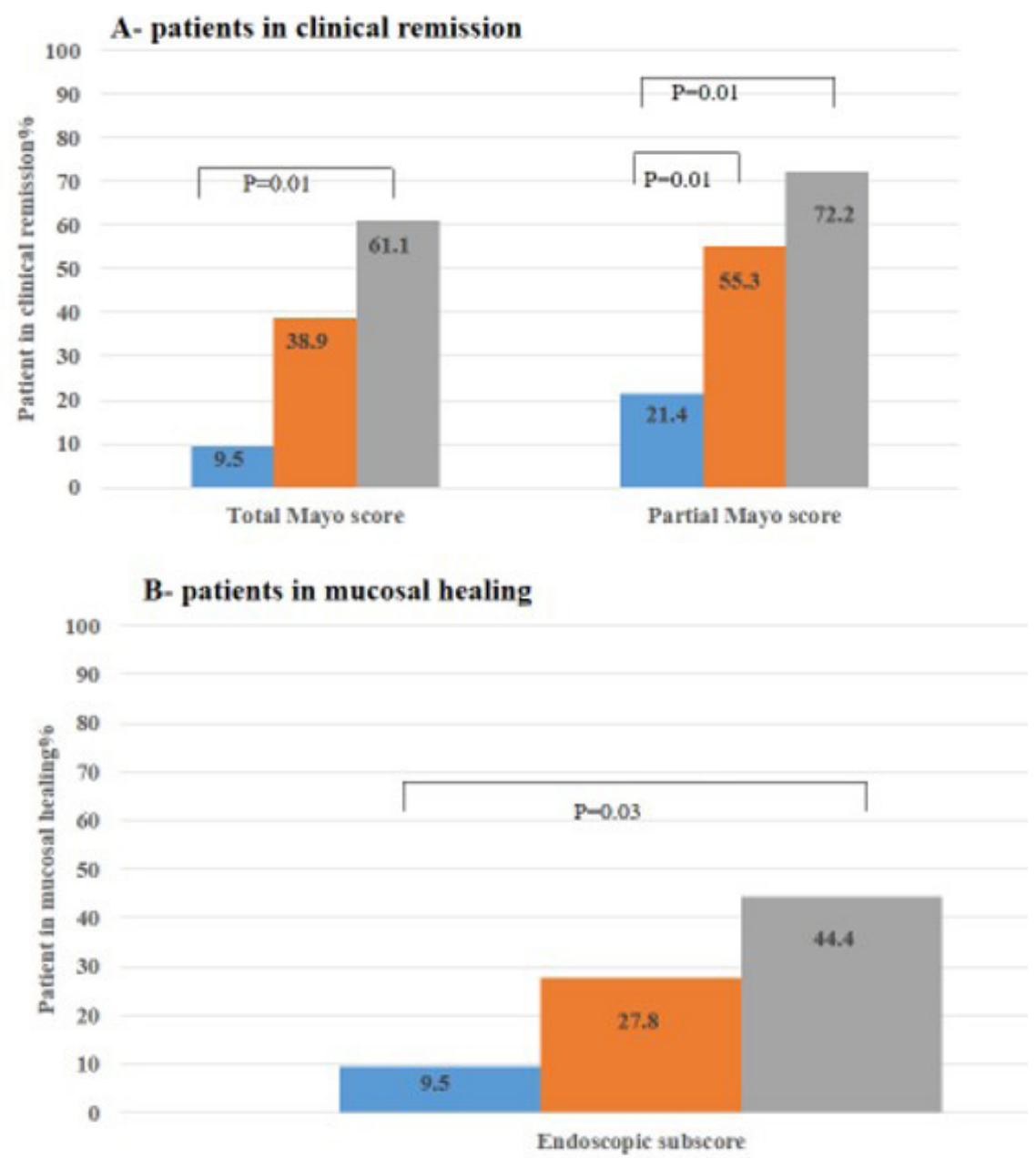

Figure 3 . Clinical Remission and Endoscopic Mucosal Healing Following 52 Weeks of Treatment with Tofacitinib. Panel A) A significant increase in percentage of patients in clinical remission based on both total Mayo score and partial Mayo score at week 52 compared to week 8. Panel B) A significant increase in percentage of patients in mucosal healing at week 52 compared to week 8.

in our population. In this study, we assessed the clinical outcomes of tofacitinib therapy in two trials of induction and maintenance therapy in patients who had active UC and failed to have desirable response to either conventional or anti-TNF therapy. We demonstrated that in this challenging patient population, tofacitinib is effective for inducing clinical response, and also maintaining the remission over one year in more than $61 \%$ of patients.

It is clear that there is a need for a systematic evaluation of the outcomes in UC in order to lessen the perceived discrepancies in regulatory decision making coming from research studies. In this study, we tried to define the outcomes based on the results of comprehensive systematic literature reviews. Using a recent published systematic review that assessed clinical trials of tofacitinib in UC, we used a definition of "clinical response" and "clinical remission" in our study which has been used by more than $77 \%$ of clinical trials addressing UC. ${ }^{17}$ Using these definitions, we found that induction therapy with tofacitinib at the FDA recommended dose for UC, produced $84 \%$ clinical response in our patients while fewer than $10 \%$ of patients were in clinical remission after induction. These results were accompanied by a significant decrease in ESR and calprotectin level. In the randomized controlled trial GEMINI 1, 8-week induction therapy with tofacitinib at a dose of $10 \mathrm{mg}$ twice daily resulted in $61 \%$ clinical remission, ${ }^{12}$ while in the OCTAVE Induction 1 and 2 trials, the remission rates at week 8 were found to be $18.5 \%$ and $16.6 \%$, respectively. ${ }^{13}$ In a later trial, the authors incorporated a more stringent definition of clinical remission which required a rectal bleeding subscore of $0 .{ }^{13}$ While our results are comparable to these studies in terms of trend of response to tofacitinib therapy and confirm its efficacy, they demonstrate lower rates of clinical remission for induction in our population. Based on a high quality study performed by Mukherjee and colleagues that evaluated the exposure-response relationships for three key efficacy endpoints of clinical 
remission, clinical response, and mucosal healing as a function of tofacitinib, the Mayo score was the most important and the only disease severity measure that negatively impacted efficacy, for two efficacy endpoints of clinical remission and mucosal healing in corrected covariate analysis. ${ }^{18}$ It is possible that the difference seen in these studies is related, at least partially, to the fact that our patients had more severe disease and higher Mayo score at the time of enrollment (10.1 vs. 8$)$. Notably, the percent of patients with anti-TNF failure in our study was higher than the GEMINI 1 trial (69.8\% vs. 18\%) which again highlights that our study population consisted of more medically refractory patients.

The large phase III OCTAVE program reported a clinical remission rate of $40.6 \%$ after one year of tofacitinib therapy - a rate that was lower than what we found in our study (61.1\%). Moreover, in our study, as previously demonstrated in clinical trials, previous failure of biologic therapy did not affect the response to treatment. ${ }^{19}$ It is possible that a combination of factors including the use of concomitant medication (for example, more than $90 \%$ of our patients had concomitant consumption of oral aminosalicylates), slow prolonged tapering of corticosteroid during maintenance therapy and study design (single-arm and site investigator) is responsible for the higher rate of clinical remission seen in our study.

In this study, we found no treatment limiting safety issues with tofacitinib and its tolerability was generally favorable among our patients. Most AEs were mild to moderate in intensity and needed no pharmacological intervention. In most clinical trials, upper respiratory tract infections and influenza-like symptoms as well as changes in lipid profile are among the most commonly reported AEs following tofacitinib therapy. ${ }^{19}$ However, in our study, bloating and excess gas were the most frequent complaints of the patients. Although we cannot rule out or rule in the relation between tofacitinib therapy and bloating, it is possible that patients more frequently reported the IBS like symptoms including excess gas following amelioration of the IBD condition. In addition to upper respiratory tract infection, $8 \%$ of our patients reported a non-itchy reddish skin rash that developed temporally close to initiation of tofacitinib. To our knowledge, this is the first time that this complication is reported following tofacitinib treatment in UC trials, although the studies which evaluated the side effects of tofacitinib in RA patients reported relative risk of skin rash close to $0.4 .^{20}$

In this study, we reported thromboembolic events in two male patients who were on maintenance therapy. Of these, only one patient had venous thromboembolism risk factors (heavy smoker and being a taxi driver). They were younger than 50 years, they were not overweight and did not have any metabolic disorders, and neither were using concomitant corticosteroids. Studies showed that in addition to UC, treatment with tofacitinib could increase the risk of venous thromboembolism events. In a posthoc analysis of patients from the tofacitinib UC clinical trial, an overall incidence rate of $0.04(0.00-0.23)$ for deep vein thrombosis was detected in the tofacitinib-treated cohort. ${ }^{21}$ Our observation regarding the occurrence of thromboembolic events following tofacitinib therapy, even in the absence of apparent venous thromboembolism risk, is in accordance with previous studies and of particular importance for future observational studies on drug safety, especially with the increasing availability of this medication for UC patients in our country.

One challenging point that may discourage patients from enrolling in clinical trials of UC is the need for performing multiple endoscopies at the time of randomization and primary endpoint. In this study, we reported the rate of clinical remission based on both total and partial Mayo score. We found that the highest rate of clinical remission was achieved when it was defined based on the partial Mayo score (72.2\%). Incorporating the invasive component of endoscopic appearance in the scoring system based on the total Mayo score definition decreased the percentage of patients achieving clinical remission to $61.1 \%$. However, more importantly, there was correlation between these two scores with regard to clinical remission in maintenance therapy. In fact, the partial Mayo score uses the non-invasive components of the full score that basically correlates to patient perceptions of response to treatment. ${ }^{19}$ In a study by Higgins and colleagues, it was shown that endoscopy items have a correlation with stool frequency and stool blood items reported by patients and it was suggested that endoscopy items may add modest additional information to indices of disease activity in UC. ${ }^{22}$ Similarly, Lewis et al found a strong correlation between the partial and total Mayo score in UC patients. ${ }^{23}$ Taking together, our results confirm the observation from previous studies and could emphasize that information derived from the invasive test of colonoscopy in fact measures the same intrinsic disease activity factors that are measured by non-invasive indices. These observations may have a practical value in a way that applying the non-invasive indices of disease activity in clinical research could lessen the cost and improve the participation of subjects.

There are several limitations to our study. First, our study was not designed as a blind randomized controlled clinical trial, and therefore, the changes seen after the intervention cannot be exclusively associated with the intervention. Second, our study lacks the data on patients who failed to respond to induction treatment as we assessed the effect of tofacitinib in patients who clinically responded to induction therapy. Also, we had some limitations in our analysis since we had a relatively small sample size from a single clinical trial that focused on patients with moderately-to-severely active disease. Moreover as colonoscopy is usually an undesirable procedure for patients, we had some colonoscopy missing data in this regard, especially in week 24 .

In conclusion, our study is the first report on the effect of tofacitinib for induction and maintenance in 
moderate-to-severe UC in a tertiary center in our region. We demonstrate acceptable efficacy and favorable safety and tolerability of this medication in medically resistant patients with no reported serious adverse events. We also produced some evidence suggesting a correlation between disease activity score that is based on the total Mayo score with partial Mayo score.

\section{Authors' Contribution}

$\mathrm{MJ}$ and HV conceived and designed the original protocol. SA, MJ and SN coordinated the study, enrolled the patients and performed the follow-up visits. SA collected, entered and analyzed all the data. MJ and SA wrote the first draft of the manuscript. SA wrote the final draft of manuscript. HV supervised the study and interpreted the data.

\section{Conflict of Interest Disclosures}

The authors declare no conflict of interest related to this work

\section{Ethical Statement}

All procedures performed in studies involving human participants were in accordance with the ethical standards of the institutional and research committee and with the 1964 Helsinki Declaration. The Institutional Review Board and local Ethics Committee of Tehran University of Medical Sciences approved this study (IR.TUMS.DDRI.REC.1397.016). All participants gave informed written consent. The trial is registered with IRCT (IRCT20181217042020N2).

\section{References}

1. Collaborators GBDIBD. The global, regional, and national burden of inflammatory bowel disease in 195 countries and territories, 1990-2017: a systematic analysis for the Global Burden of Disease Study 2017. Lancet Gastroenterol Hepatol. 2020;5(1):17-30. doi: 10.1016/S2468-1253(19)30333-4.

2. Malekzadeh MM, Vahedi H, Gohari K, Mehdipour P, Sepanlou SG, Ebrahimi Daryani N, et al. Emerging Epidemic of Inflammatory Bowel Disease in a Middle Income Country: A Nation-wide Study from Iran. Arch Iran Med. 2016;19(1):2-15.

3. Malekzadeh MM, Sima A, Alatab S, Sadeghi A, Daryani NE, Adibi P, et al. Iranian Registry of Crohn's and Colitis: study profile of first nation-wide inflammatory bowel disease registry in Middle East. Intest Res. 2019;17(3):330-9.

4. Ungaro R, Mehandru S, Allen PB, PeyrinBiroulet L, Colombel J-F. Ulcerative colitis. Lancet. 2017;389(10080):1756-70. doi: 10.5217/ir.2018.00157.

5. Neurath MF. Cytokines in inflammatory bowel disease. Nat Rev Immunol. 2014;14(5):329-42. doi: 10.1038/nri3661.

6. Salas A, Hernandez-Rocha C, Duijvestein M, Faubion W, McGovern D, Vermeire S, et al. JAK-STAT pathway targeting for the treatment of inflammatory bowel disease. Nat Rev Gastroenterol Hepatol. 2020;17(6):323-337. doi: 10.1038/s41575-020-0273-0.

7. Schreiner P, Neurath MF, Ng SC, El-Omar EM, Sharara AI, Kobayashi T, et al. Mechanism-Based Treatment Strategies for IBD: Cytokines, Cell Adhesion Molecules, JAK Inhibitors, Gut Flora, and More. Inflamm Intest Dis. 2019;4(3):79-96. doi: 10.1159/000500721.

8. Villarino AV, Kanno Y, O’Shea JJ. Mechanisms and consequences of Jak-STAT signaling in the immune system.
Nat Immunol. 2017;18(4):374-384. doi: 10.1038/ni.3691.

9. Hedl M, Proctor DD, Abraham C. JAK2 Disease-Risk Variants Are Gain of Function and JAK Signaling Threshold Determines Innate Receptor-Induced Proinflammatory Cytokine Secretion in Macrophages. J Immunol. 2016;197(9):3695-3704. doi: 10.4049/jimmunol.1600845.

10. Huang H, Fang M, Jostins L, Umicevic Mirkov M, Boucher $\mathrm{G}$, Anderson CA, et al. Fine-mapping inflammatory bowel disease loci to single-variant resolution. Nature. 2017;547(7662):173-8. doi: 10.1038/nature22969.

11. Liu JZ, van Sommeren S, Huang H, Ng SC, Alberts R, Takahashi A, et al. Association analyses identify 38 susceptibility loci for inflammatory bowel disease and highlight shared genetic risk across populations. Nat Genet. 2015;47(9):979-986. doi: 10.1038/ng.3359.

12. Sandborn WJ, Ghosh S, Panes J, Vranic I, Su C, Rousell S, et al. Tofacitinib, an oral Janus kinase inhibitor, in active ulcerative colitis. N Engl J Med. 2017;376(18):1723-1736. doi: 10.1056/NEJMoa1606910.

13. Sandborn WJ, Su C, Sands BE, D'Haens GR, Vermeire $S$, Schreiber S, et al. Tofacitinib as Induction and Maintenance Therapy for Ulcerative Colitis. N Engl J Med. 2017;376(18):1723-1736. doi: 10.1056/NEJMoa1606910.

14. D’Haens G, Sandborn WJ, Feagan BG, Geboes K, Hanauer $\mathrm{SB}$, Irvine EJ, et al. A review of activity indices and efficacy end points for clinical trials of medical therapy in adults with ulcerative colitis. Gastroenterology. 2007;132(2):76386. doi: 10.1053/j.gastro.2006.12.038.

15. Sandborn WJ, Panes J, Zhang H, Yu D, Niezychowski W, Su C. Correlation Between Concentrations of Fecal Calprotectin and Outcomes of Patients With Ulcerative Colitis in a Phase 2 Trial. Gastroenterology. 2016;150(1):96102. doi: 10.1053/j.gastro.2015.09.001.

16. Varyani F, Argyriou K, Phillips F, Tsakiridou E, Moran GW. Profile of Tofacitinib in the Treatment of Ulcerative Colitis: An Evidence-Based Review of Recent Data. Drug Des Devel Ther. 2019;13:4091-4105. doi: 10.2147/DDDT.S182891

17. Lohan C, Diamantopoulos A, LeReun C, Wright E, Bohm $\mathrm{N}$, Sawyer LM. Tofacitinib for the treatment of moderately to severely active ulcerative colitis: a systematic review, network meta-analysis and economic evaluation. BMJ Open Gastroenterol. 2019 Jul 30;6(1):e000302. doi: 10.1136/bmjgast-2019-000302. Erratum in: BMJ Open Gastroenterol. 2020;7(1):e000302corr1

18. Mukherjee A, Hazra A, Smith MK, Martin SW, Mould DR, Su C, et al. Exposure-response characterization of tofacitinib efficacy in moderate to severe ulcerative colitis: Results from a dose-ranging phase 2 trial. Br J Clin Pharmacol. 2018;84(6):1136-1145. doi: 10.1111/bcp.13523.

19. Weisshof R, Aharoni Golan M, Sossenheimer PH, El Jurdi K, Ollech JE, Pekow J, et al. Real-World Experience with Tofacitinib in IBD at a Tertiary Center. Dig Dis Sci. 2019;64(7):1945-1951. doi: 10.1007/s10620-019-05492-y.

20. He Y, Wong AYS, Chan EW, Lau WCY, Man KKC, Chui CSL, et al. Efficacy and safety of tofacitinib in the treatment of rheumatoid arthritis: a systematic review and metaanalysis. BMC Musculoskelet Disord. 2013;14:298. doi: 10.1186/1471-2474-14-298.

21. Sandborn WJ, Panés J, Sands BE, Reinisch W, Su C, Lawendy $\mathrm{N}$, et al. Venous thromboembolic events in the tofacitinib ulcerative colitis clinical development programme. Aliment Pharmacol Ther. 2019;50(10):1068-1076. doi: 10.1111/ 
apt.15514.

22. Higgins PD, Schwartz M, Mapili J, Zimmermann EM. Is endoscopy necessary for the measurement of disease activity in ulcerative colitis? Am J Gastroenterol. 2005;100(2):35561. doi: 10.1111/j.1572-0241.2005.40641.x.
23. Lewis JD, Chuai S, Nessel L, Lichtenstein GR, Aberra FN, Ellenberg JH. Use of the noninvasive components of the Mayo score to assess clinical response in ulcerative colitis. Inflamm Bowel Dis. 2008;14(12):1660-6. doi: 10.1002/ ibd. 20520

(c) (i) 2021 The Author(s). This is an open-access article distributed under the terms of the Creative Commons Attribution License (http://creativecommons. org/licenses/by/4.0), which permits unrestricted use, distribution, and reproduction in any medium, provided the original work is properly cited. 